\title{
Impact of pregnancy on prognosis of differentiated thyroid cancer: clinical and molecular features
}

\author{
Ilaria Messuti ${ }^{*}$, Stefania Corvisieri ${ }^{*}$, Francesca Bardesono, Ida Rapa', \\ Jessica Giorcelli ${ }^{1}$, Riccardo Pellerito ${ }^{2}$, Marco Volante ${ }^{1}$ and Fabio Orlandi \\ Endocrine Unit, Department of Oncology, Presidio Sanitario Gradenigo, University of Turin, \\ Corso Regina Margherita 10, Turin, Italy, ${ }^{1}$ Pathology Unit, Department of Oncology, San Luigi Hospital, \\ University of Turin, Orbassano, Turin, Italy and ${ }^{2}$ Nuclear Medicine Unit, Mauriziano Hospital, Turin, Italy \\ *(I Messuti and S Corvisieri contributed equally to this work)
}

Correspondence should be addressed to F Orlandi

Email

fabio.orlandi@unito.it

\begin{abstract}
Objective: Differentiated thyroid cancer (DTC) commonly occurs in women of child-bearing age and represents the second most frequent tumor diagnosed during pregnancy only behind breast cancer. It is possible that associated physiological changes could favor tumor development and growth. However, few data are available about the outcome of DTC related to pregnancy, leading to conflicting results.

Methods: Among the study population, 340 patients with DTC $<45$ years old were retrospectively studied. Patients were divided into three groups according to the time of tumor diagnosis in respect of pregnancy. Group 1, diagnosis of DTC at least 2 years after delivery; group 2, diagnosis during pregnancy or within the second year after delivery; and group 3, nulliparous patients at the time of diagnosis. We evaluated clinical outcome and immunohistochemical expression of estrogen receptor $\alpha$ $(E R \alpha), E R \beta$, progesterone receptor, and aromatase. We also analyzed the gene expression of NIS (SLC5A5) and the prevalence of $B R A F^{\mathrm{V} 600 \mathrm{E}}$ mutations.

Results: Persistence/recurrence of disease was significantly higher in group 2 patients than control groups $(P=0.023)$. No significant differences were observed in other clinical parameters. Furthermore, no differences among the groups were recorded about ER pattern, NIS expression, and BRAF mutations.

Conclusions: Persistence/recurrence of DTC is significantly higher in pregnant patients, suggesting that pregnancy could really exert a negative prognostic role in patients with DTC. The underlying mechanisms are not yet clarified and further studies are required. Our results suggest that a more careful follow-up is needed when diagnosis of DTC occurs during pregnancy or shortly after.
\end{abstract}

\section{Introduction}

Differentiated thyroid cancer (DTC) is a relatively rare neoplasia. It represents $3.6 \%$ of all malignant tumors in the USA (SEER Cancer Statistics Review, National Cancer Institute Surveillance, Epidemiology, and End Results; 1975-2005, available from http://seer.cancer.gov) and it is generally characterized by good prognosis. Consequently, studies evaluating the prognosis of this tumor have to consider a wide number of cases and a long-term follow-up to highlight the differences in survival or disease recurrence rate. The majority of relapses usually occurs within 5 years from the initial treatment, and only sporadic cases have been subsequently documented $(1,2)$. Despite the low incidence, in the USA, DTC represents the second most frequently diagnosed tumor
(C) 2014 The authors Published by Bioscientifica Ltd

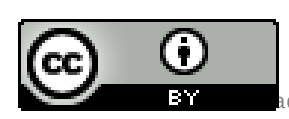

This work is licensed under a Creative Commons Attribution 3.0 Unported License. 
during pregnancy, only after breast cancer. In women of child-bearing age, about $10 \%$ of thyroid carcinomas are diagnosed during pregnancy or early after delivery (SEER Cancer Statistics Review, National Cancer Institute Surveillance, Epidemiology, and End Results; 1975-2005, available from http://seer.cancer.gov). These findings have led us to hypothesize that during this period the presence of several physiologic changes, such as hormonal secretion, growth factors, and negative iodine balance, could create a favorable environment for the development and growth of tumors.

However, only a few studies about the outcome of DTC related to pregnancy have been published. A recent review (3) has reported that pregnancy is not generally described in literature as a determining condition for prognosis of DTC, neither in terms of DTC-related death (4), nor of overall survival $(5,6)$.

Nevertheless, these findings are in contrast with the more recent study published by Vannucchi et al. (7), who reported that DTC in pregnant women had a significant increase of persistent/recurrent disease than those in nonpregnant patients. Since the parameters and the methodology in each study were very different, their results were not easily comparable. In fact, the studies conducted by Yasmeen et al. (5) and Herzon et al. (6) focused mainly on the overall survival, while the study of Moosa \& Mazzaferri (4) had DTC-related death and disease recurrence, evaluated by biopsy or by 131-I uptake in distant sites, as primary focus. On the contrary, Vannucchi et al. (7) have evaluated persistent/recurrent DTC through more sensitive tests, such as basal and stimulated tireoglobuline (Tg) levels after exogenous thyroid-stimulating hormone (TSH) injection (recombinant human TSH (rh-TSH), Thyrogen, Genzyme Corporation, Sanofi Company, Cambridge, MA, USA), which have not been used in the other studies and may - at least partially - explain the different conclusions.

Moreover, Vannucchi et al. (7) observed a significantly higher immunohistochemical expression of the estrogen receptor $\alpha(\mathrm{ER} \alpha)$ in tumors sampled from pregnant women, compared with the control groups. With special reference to hormone receptor expression in thyroid tumors, a recent study (8) on the immunohistochemical expression of ER and androgen receptor (AR) in DTC showed that ER $\alpha$ was acquired or increased in tumor samples as compared with the corresponding normal tissue, whereas AR and ER $\beta$ expression was decreased in tumors compared with the surrounding normal tissue. These patterns appeared also to be associated with the clinical behavior, being the high expression of ER $\alpha$ and AR and the low expression of $\operatorname{Er} \beta$ associated with a more aggressive phenotype.

In such a controversial situation, we therefore designed the present study to characterize at clinical, phenotypical, and molecular levels DTC cases in pregnancy as compared with matched control groups.

\section{Subjects and methods}

\section{Patients}

We retrospectively evaluated more than 1200 medical records of patients with DTC treated and followed-up from 2001 to 2011 at the Nuclear Medicine Department of Mauriziano Hospital, which covers up to $80 \%$ of all radioiodine ablations with I-131 (RAI) performed in the Piedmont region. This allowed us to obtain an extremely homogeneous population, representative of DTC epidemiology in the fertile women population.

Among them, 340 women were selected according to the following inclusion criteria:

- age $\leq 45$ years at the time of surgery

- total thyroidectomy

- I-131 radioiodine ablation

- Levo thyroxine (L-T $\left.{ }_{4}\right)$ TSH-suppressive therapy $(\mathrm{TSH} \leq 0.1 \mathrm{mU} / \mathrm{l})(9)$

- follow-up $\geq 1$ year

- rh-TSH test during follow-up or persistent disease (Tg measured under suppressive therapy with $\mathrm{L}_{-} \mathrm{T}_{4}$ $(\mathrm{S}-\mathrm{Tg})>2 \mathrm{ng} / \mathrm{ml})$

Patients were divided into three groups according to the time of diagnosis of DTC with respect to pregnancy. Group 1 included women $(n=152$, median age 40 , range 25-45) with diagnosis of DTC at least 2 years after delivery. Group 2 included women $(n=38$, median age 35 , range 26-41) with diagnosis of DTC during pregnancy or within 2 years after delivery. Group 3 included nulliparous patients at the time of diagnosis ( $n=150$, median age 30 , range $15-45)$.

Tumors were classified following the World Health Organization classification (10), staged according to the 6th edition of TNM staging (American Joint Committee on Cancer (AJCC)) (11), and classified as low and high risk according to the European Consensus Statement criteria (9). ETA guidelines divide patients into three groups: very low, low, and high risk, but the first group was not represented in our series because it includes patients with no indications for RAI. 
Remission or persistent/recurrent disease was defined according to the European and American guidelines for the management of DTC $(9,12)$ :

- remission: $\mathrm{S}-\mathrm{Tg}$ and $\mathrm{Tg}$ measured after stimulation with rh-TSH (rh-TSH-Tg) $<0.6 \mu \mathrm{g} / \mathrm{l}$, negative anti-Tg antibody (AbTg), and normal neck ultrasound.

- persistent/recurrent disease: at least one of the following criteria:

- S-Tg $>2 \mu \mathrm{g} / \mathrm{l}$.

- rh-TSH-Tg $>2 \mu \mathrm{g} / \mathrm{l}$.

- persistence of AbTg $>4$ years with a trend to increase (an increasing antibody production or new antibodies appearance as a consequence of an increase in autoantigen production) $(13,14)$

- neck or distant metastasis

- radioiodine uptake outside thyroid bed.

Serum Tg levels were measured during $\mathrm{L}^{-\mathrm{T}_{4}}$ withdrawal, immediately before RAI and after 12 months of $\mathrm{L}_{-} \mathrm{T}_{4}$ suppressive therapy. Then, patients received one injection of rh-TSH $(0.9 \mathrm{mg}$ i.m., Thyrogen, Genzyme Corporation, Sanofi Company) for 2 consecutive days; serum samples for TSH and Tg measurements were collected on day 0 (before first rh-TSH administration), day 3 , and day 4 . Neck ultrasonography was performed 6 and 12 months after RAI. TSH levels were evaluated using a chemiluminescent immunoassay (Access Immunoassay Systems, Beckman Coulter, Inc., Brea, CA, USA). Tg levels were determined using chemiluminescent immunoassay (Access Immunoassay Systems, Beckman Coulter, Inc.), with a functional sensitivity of $0.6 \mu \mathrm{g} / \mathrm{l}$; $\mathrm{AbTg}$ were detected with chemiluminescent immunoassay (Access Immunoassay Systems, Beckman Coulter, Inc.). On the basis of Tg assay, we considered $0.6 \mu \mathrm{g} / \mathrm{l}$ as the cutoff value between undetectable and measurable Tg levels, according to Mazzaferri et al. (1).

\section{Immunohistochemical analysis of hormone receptors and aromatase in tumor tissues}

Immunohistochemical evaluation of $\mathrm{ER} \alpha, \mathrm{ER} \beta$, progesterone receptor (PGR), and aromatase was performed in 37 histological specimens selected from the three different groups (12 of group 1, ten of group 2, and 15 of group 3). The cases for immunohistochemical analysis were blinded, selected to obtain three groups homogenous in terms of age and stage, regardless of the outcome.

Immunohistochemical analyses were carried out on paraffin-embedded tissue sections of $5 \mu \mathrm{m}$, after dewaxing, dehydration in alcohol, and rehydration in PBS, pH 7.5.
Endogenous peroxidase block was performed through immersion of the slides in $0.3 \%$ solution of methanol and hydrogen peroxide for $15 \mathrm{~min}$. Then, the sections were incubated with the following monoclonal primary antibodies: ER $\alpha$ (clone 1D5, dilution 1:300, Dako, Glostrup, DK), ER $\beta$ (clone PPG5/10, dilution 1:50, Dako), PGR (clone 636, dilution 1:300, Dako), and aromatase (clone mca2077s, dilution 1:50, Serotec, Kidlington, UK). A biotin-free, dextran chain-based detection system (EnVysion, Dako) and diaminobenzidine as the chromogen were used according to standard protocols. All markers were assessed in tumoral and peritumoral tissues using $H$-score evaluation, which takes into account both quantitative and qualitative expression with a 0-300 range scale.

\section{Molecular analysis}

Nucleic acids isolation $>$ Genomic DNA was isolated from formalin-fixed, paraffin-embedded tissues using QIAamp DNA Mini Kit (Qiagen). RNA was isolated from paraffin-embedded material using the high pure RNA paraffin kit (Roche), following the manufacturer's instructions. The quantity of isolated DNA and RNA was assessed using a Biophotometer (Eppendorf, Hamburg, Germany).

BRAF point mutation analysis $~-$ The presence of $B R A F$ point mutation (V600E) was analyzed using pyrosequencing and PCR primers following previously published protocols (15). PCR amplification for the pyrosequencing assay was carried out according to standard protocols. The amplicons were mixed with sequencing primers and sequencing was performed using a PyroGold Reagent Kit (Biotage AB, Uppsala, Sweden) according to the manufacturer's protocol. Results were analyzed using the PSQ-96 MA 2.0.2 Software (Biotage AB).

Quantitative real-time PCR for the sodium/iodide symporter $>$ Relative cDNA quantitation of the sodium/ iodide symporter (NIS; SLC5A5) and an internal reference gene ( $\beta$-actin) were done using a fluorescencebased real-time detection method (ABI PRISM 7900 Sequence Detection System, TaqMan; Applied Biosystems/ Life Technologies). $\beta$-actin primers and probe were previously published (16), whereas for NIS the TaqMan gene expression assay $20 \times$ (SLC5A5 Hs00166567_m1, Applied Biosystems) was used according to the manufacturer's instructions. The PCR mixture consisted of $1200 \mathrm{nmol} / \mathrm{l}$ of each primer, $200 \mathrm{nmol} / \mathrm{l}$ probe, $200 \mathrm{nmol} / \mathrm{l}$ each of dATP, dCTP, dGTP, dTTP, $3.5 \mathrm{mmol} / \mathrm{l}$ $\mathrm{MgCl}_{2}$, and $1 \times$ TaqMan Universal PCR Master Mix to 
a final volume of $20 \mu \mathrm{l}$ (all reagents were from PE Applied Biosystems). Cycling conditions were $50^{\circ} \mathrm{C}$ for $2 \mathrm{~min}$, $95^{\circ} \mathrm{C}$ for $10 \mathrm{~min}$, followed by 46 cycles at $95^{\circ} \mathrm{C}$ for $15 \mathrm{~s}$ and $60{ }^{\circ} \mathrm{C}$ for $1 \mathrm{~min}$. To analyze target gene expression in individual tumors, the relative gene expression levels were expressed as ratios (differences between the $C_{t}$ values) between two absolute measurements (genes of interest/ internal reference gene). Then, the $\Delta \Delta C_{t}$ values were calculated subtracting $\Delta C_{\mathrm{t}}$ values of each case to the value of the normal sample expression, and converting the ratio by the $2^{-\Delta \Delta C_{\mathrm{t}}}$ formula; cases were considered of low or high expression according to the median expression level obtained.

\section{Statistical analysis}

The clinical (age, outcome, number of treatments, ablation-Tg levels, and high/low risk classification) and pathological/molecular features (histology, pTNM stage, hormone receptor expression, NIS gene expression, and $B R A F$ mutation status) were compared among the three groups of patients by using the $\chi^{2}$-test for dichotomic variables and the Mann-Whitney $U$ and Kruskal-Wallis tests for continuous variables, as appropriate. The reciprocal correlation among immunohistochemical markers was evaluated using the Spearman's test. Statistical significance was defined as $P<0.05$.

A logistic multivariable analysis was performed. Dependent dichotomous variable was tumor persistence/recurrence (1) or remission (0). Age, T, N, and multifocality of primary tumor and pregnancy (DTC diagnosis during pregnancy or within 2 years after delivery: 1; other groups: 0) were the independent variables. All these analyses were performed using STATISTICA for Windows, ver. 8.0.

\section{Results}

Clinical, biochemical, histopathological, and molecular parameters in the three groups are reported in Table 1. No significant differences were noticed in the number of treatments for achieving clinical remission, in the tumor size or extrathyroidal invasion, in the lymphnodal metastatic involvement at diagnosis, in histology, and in high risk/low risk classification of patients according to the ETA guidelines (9).

Clinical remission was obtained in $150 / 152$ patients $(98.7 \%)$ of group $1,34 / 38$ patients $(89.5 \%)$ of group 2 ,

Table 1 Clinical, histological, and molecular characteristics of patients with a DTC diagnosis at least 2 years after delivery (group 1), during pregnancy or within 2 years after delivery (group 2), or before pregnancy/nulliparous (group 3).

\begin{tabular}{l}
\hline \\
\hline Age at diagnosis (years): median (range) \\
Duration of follow-up (years): median \\
(range) \\
Remission \\
Persistence/recurrence \\
Number of treatments (average) \\
Ablation-HTG \\
$<10 \mathrm{ng} / \mathrm{ml}^{\mathrm{b}}$ \\
$>10 \mathrm{ng} / \mathrm{ml}^{\mathrm{b}}$ \\
High risk \\
Low risk \\
TNM \\
$\mathrm{T}<3$ \\
$\mathrm{~T}>3$ \\
$\mathrm{~N}-$ \\
$\mathrm{N}+$ \\
Histology \\
$\mathrm{High}$ risk \\
Low risk \\
ER $\alpha$ tumor expression \\
ER $\beta$ tumor expression \\
$\mathrm{PGR}$ tumor expression \\
$B R A F^{V 600 \mathrm{E}}$ mutation \\
$\mathrm{NIS}$ fold change $<1$ \\
\end{tabular}

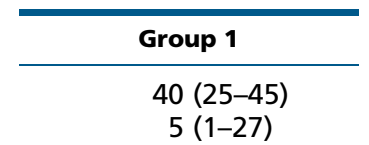
$150 / 152(98.7 \%)$
$2 / 152(1.3 \%)$
1.19

$127 / 152(83.5 \%)$

$25 / 152(16.5 \%)$

$68 / 152(44.7 \%)$

$84 / 152(55.3 \%)$

$105 / 152(69 \%)$

$47 / 152(31 \%)$

$114 / 152(75 \%)$

$38 / 152(25 \%)$

$54 / 152(35.5 \%)$

$98 / 152(64.5 \%)$

$3 / 12(25 \%)$

$5 / 12(41.7 \%)$

$4 / 12(33.3 \%)$

$3 / 12(25 \%)$

$8 / 12(66.6 \%)$

\begin{tabular}{c}
\hline Group 2 \\
\hline $35(26-41)$ \\
$6(1-10)$ \\
\\
$34 / 38(89.5 \%)$ \\
$4 / 38(10.5 \%)$ \\
1.21 \\
$27 / 38(71 \%)$ \\
$11 / 38(29 \%)$ \\
$19 / 38(50 \%)$ \\
$19 / 38(50 \%)$ \\
$26 / 38(68.4 \%)$ \\
$12 / 38(31.6 \%)$ \\
$29 / 38(76.3 \%)$ \\
$9 / 38(23.6 \%)$ \\
$16 / 38(42.1 \%)$ \\
$22 / 38(57.9 \%)$ \\
$3 / 10(30 \%)$ \\
$5 / 10(50 \%)$ \\
$3 / 10(30 \%)$ \\
$4 / 9(44.4 \%)$ \\
$5 / 8(62.5 \%)$ \\
\hline
\end{tabular}

\begin{tabular}{c}
\hline Group 3 \\
\hline $30(15-45)$ \\
$6(1-20)$
\end{tabular}

\begin{tabular}{c}
\hline $\boldsymbol{P}$ value \\
\hline$<0.001$ \\
0.31
\end{tabular}

$143 / 150(95.3 \%)$

$7 / 15(4.7 \%)$

1.28

$0.023^{\mathrm{a}}$

0.22

$110 / 150(73.3 \%)$

40/150 (26.7)

$79 / 150(52.7 \%)$

$71 / 150(47.3 \%)$

0.060

0.38

$95 / 150(63.3 \%)$

$55 / 150(36.7 \%)$

$102 / 150(68 \%)$

$48 / 150(32 \%)$

0.85

$62 / 150(41.3 \%)$

$88 / 150(58.7 \%)$

$4 / 14(28.6 \%)$

$7 / 15(46.7 \%)$

$8 / 15(53.3 \%)$

$9 / 15(60 \%)$

$9 / 13(69.2 \%)$
0.54

0.85

0.96

0.92

0.419

0.19

0.9

${ }^{\mathrm{a} G r o u p} 2$ was significantly different as compared with both groups 1 and 3 .

${ }^{\mathrm{b}}$ The ablation-HTG cut-off was defined according to Webb et al. (17). 
and in $143 / 150$ patients (95.3\%) of group 3. Persistent/ recurrent disease was observed in $2 / 152$ patients $(1.3 \%)$ of group $1,4 / 38$ patients $(10.5 \%)$ of group 2 , and in $7 / 150$ patients $(4.7 \%)$ of group 3. Our results showed a significant difference $\left(\chi^{2}, 7.532 ; P=0.023\right)$ in the outcome among the three groups, with a greater percentage of persistent disease in group 2 than in groups 1 and 3 . Groups 1 and 3 did not show any significant difference. Only 4/38 patients in group 2 had cytological diagnosis while pregnant. They underwent thyroidectomy in the early postpartum period, achieving clinical remission, showing that the surgical delay of a few months was not a factor that could influence the worst outcome of group 2.

With regards to the expression of hormone receptors (Fig. 1), the percentage of intratumoral and peritumoral expression of ER $\alpha$ in the 37 histological samples was globally low, with no detection of significant differences between the groups $(P=0.96)$. ER $\beta$ showed a high expression in the peritumoral tissue in a large number of cases, while in tumoral tissue its expression was quite variable, similarly in the three groups $(P=0.82)$. PGR expression was mostly negative in peritumoral tissue, while it was quite variable in tumoral tissue, in a similar way in the three groups $(P=0.41)$. A significant correlation was observed in tumor tissues between ER $\alpha$ and PGR (Spearman's $R$ value, $R=0.49$; $P=0.002$ ). Aromatase expression was found to be negative both on peritumoral and tumoral tissue in all the samples analyzed. $B R A F^{\mathrm{V} 600 \mathrm{E}}$ mutation, known as a negative prognostic factor (18), was detected in $25 \%$ in group 1 , $44.4 \%$ in group 2 , and $60 \%$ in group 3 (average of whole samples $=43 \%)$. The difference was not statistically significant $(P=0.191)$, showing that the worst outcome observed in the patients of group 2 is independent from $B R A F$ mutation. However, $B R A F$ was mutated in $100 \%$ of patients
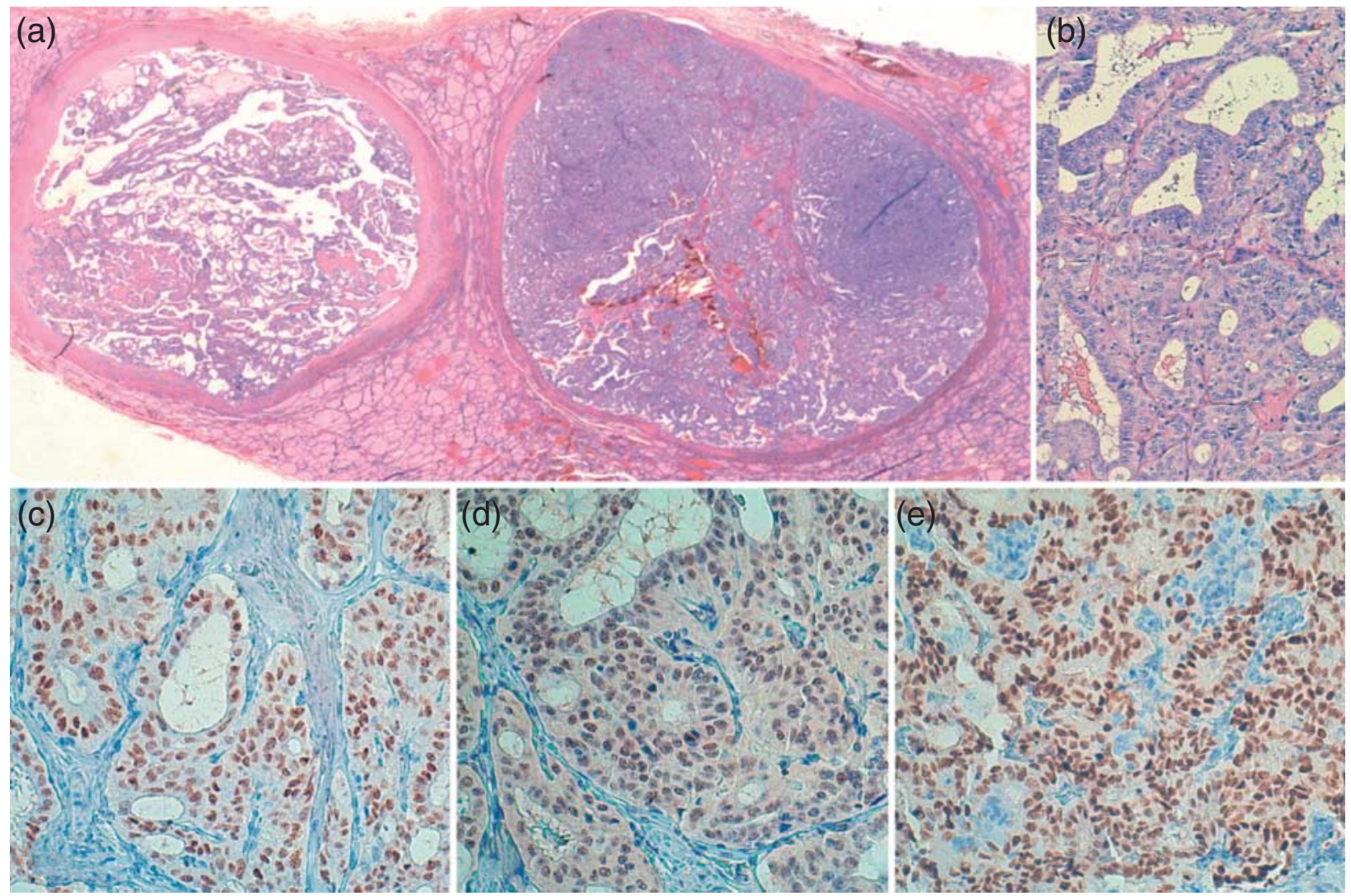

\section{Figure 1}

Immunohistochemical analysis of hormone receptors. A case of multifocal papillary carcinoma (group 3) ((a) H\&E, original magnification $40 \times)$, with the predominant nodule of the follicular variant ((b) $H \& E$, original magnification $200 \times)$, and high expression of ER $\alpha$ (c), ER $\beta$ (d), and PGR (e) ((c, d and e) immunoperoxidase, original magnification $200 \times$ ). 
Table 2 Logistic regression analysis for persistence/recurrence of DTC.

\begin{tabular}{l}
\hline Variable \\
\hline Pregnancy \\
Er $\beta$-positive staining \\
Presence of BRAF mutation \\
High NIS expression
\end{tabular}

\begin{tabular}{|c|c|}
\hline $\mathbf{R R}(95 \% \mathrm{Cl})$ & $P$ value \\
\hline $1.26(0.97-1.55)$ & 0.09 \\
\hline $0.69(0.38-1.00)$ & 0.06 \\
\hline $1.46(1.16-1.77)$ & 0.005 \\
\hline $0.66(0.36-0.96)$ & 0.03 \\
\hline
\end{tabular}

with persistence of disease and in $37.5 \%$ of patients in remission, irrespective of the group.

NIS gene expression levels were also not different in the three groups $(P=0.82)$ nor associated with $B R A F$ mutation status $(P=0.55)$.

Logistic multivariable analysis performed on the whole population (thus excluding molecular analyses) showed pregnancy (group 2) as the unique independent variable for persistent/recurrent DTC prediction. The relative risk (RR) was $1.12(95 \% \mathrm{CI}, 1.02-1.22 ; P=0.02)$. Age, $T, N$, and multifocality of the primary tumor did not enter the model.

In the 37 patients with molecular and immunohistochemical data available, BRAF mutation and low NIS expression were strong independent predictors of persistence/recurrence of DTC (Table 2), whereas ER $\alpha$ and PGR did not enter the model. Pregnancy and ER $\beta$ positivity were of borderline statistical significance..

Power analysis was carried out grouping the entire population into patients with DTC during or within 2 years after delivery (38 subjects) vs all other patients (302 subjects), with values of 79 and $87 \%$, by two-sided and one-sided test respectively.

\section{Discussion}

Thyroid cancer discovered during pregnancy represents a challenge for the clinicians because, at present, there are still no reliable data available supporting a specific management of pregnancy-associated DTCs. Currently, pregnant patients with a cytologically suspicious thyroid nodule for DTC do not require surgery during pregnancy except in cases of rapid nodular growth and/or the appearance of lymph node metastases (19).

Most studies showed that pregnancy did not worsen the prognosis of DTC. In four studies, the prognosis of women with DTC diagnosed either during pregnancy or within the first postpartum period was compared with that of women diagnosed at another time as controls. In three of these works $(4,5,6)$, no difference was found in DTC prognosis between pregnant women and control groups.
However, in the fourth study (7), Vannucchi et al. reported a significantly worse outcome in pregnant patients. As a matter of fact, they observed $60 \%$ of recurrent/persistent disease in pregnant women (group 2) vs $4.2 \%$ in women with DTC diagnosed more than 1 year after delivery (group 1) and 13.1\% in nulliparous patients (group 3). Moreover, a higher expression of ER $\alpha$ in tumor samples of pregnant women was reported.

In order to verify these conflicting results, we selected a homogeneous population, dividing patients into three groups according to the criteria adopted by Vannucchi et al. We extended group 2 to women with DTC diagnosis within 2 years after delivery instead of 1 year, arbitrarily assuming that in tumors with low biological aggressiveness, such as DTC, pregnancy-induced hyperestrogenism may exert its tissue activity in a longer period. To our knowledge, no published data are available on this issue. Moreover, in our population the rate of persistent/ recurrent disease in patients diagnosed within 1 year or between 1 and 2 years after delivery was very similar (9.5\%, 2/21 cases and $11.7 \%, 2 / 17$ cases respectively). However, all the patients (14/14) diagnosed between 2 and 3 years after delivery displayed clinical remission.

Consistent with the data reported by Vannucchi et al., we confirmed a significant correlation between pregnancy and a worse outcome of DTC $(P=0.023)$, representing the unique independent variable for persistent/recurrent disease prediction.

Indeed, thyroid cancer diagnosed during pregnancy (group 2) was found to be significantly associated with persistence or relapse of DTC compared with those diagnosed more than 2 years after delivery (group 1) or before pregnancy (group 3).

Taken together, recent evidence has supported the hypothesis that pregnancy may negatively affect the prognosis of DTC. The discrepancy with previous studies could be attributed to the different criteria used for the outcome evaluation, as suggested elsewhere (3). Previous papers used the overall survival, DTC-related death, and disease recurrence (evaluated by biopsy or whole-body scan) as outcome criteria, which were probably not appropriate for a long survival disease with frequent indolent course. In the present study, according to Vannucchi et al., the persistence/recurrence of disease was investigated using more sensitive and precocious markers, such as basal and rh-TSH-stimulated thyroglobulin, and neck ultrasonography, as suggested by European and American guidelines $(9,12)$.

Nevertheless, the worst outcome in patients of group 2 cannot be referred to a higher prevalence of a worse 
staging at the time of diagnosis or to a more aggressive histological phenotype because, in our study, no significant differences in the examined clinical and morphological parameters were observed.

The mechanisms by which pregnancy could affect the DTC outcome are not easily explainable. In order to verify whether molecular and/or phenotypical features influence the results above, we tested the protein expression of sex hormone receptors, as well as the gene expression of NIS and the prevalence of $B R A F$ mutations in the three groups. Indeed, we cannot support the negative prognostic role of estrogens, as previously suggested (7), considering that our results did not show any significant expression of $\mathrm{ER} \alpha$ and no differences among the three groups were observed. The discrepancy between these results has to be clarified, but a difference in the methodological approach could be considered. For example, different antibody dilutions were used in the two works (1:300 vs 1:100 dilution). However, it has to be noted that the good correlation between the low expression of ER $\alpha$ and PGR justifies the reliability of our findings. The immunohistochemical analysis was performed also for the detection of ER $\beta$, showing a variable expression without any significant difference among the three groups of patients. Furthermore, aromatase expression was generally very low, leading us to rule out its potential pathophysiological role.

In the multivariable logistic regression analysis, $B R A F^{\mathrm{V} 600 \mathrm{E}}$ mutations were associated with a worse prognosis, but their similar distribution among the groups excludes a pathophysiological role on the poorer outcome of group 2 patients.

We hypothesized that the worse outcome of group 2 could be explained by a lower response to radioiodine therapy. As shown by the multivariable logistic regression analysis, lower expression of NIS is associated with a higher persistence/recurrence of DTC, but its distribution was not different among the three groups, excluding a role in affecting the outcome of group 2 .

In conclusion, our results, obtained in a large homogeneous population, confirm that pregnancy could really exert a negative prognostic role, at least in terms of risk of persistent disease or recurrence, in patients with DTC. Further studies are needed to clarify the pathophysiological mechanisms. At the present state of our knowledge, a more careful follow-up is needed when diagnosis of DTC occurs during pregnancy or shortly after. However, the impact on DTC prognosis is not so heavy to justify the reconsideration of the American guidelines for the management of thyroid cancer during pregnancy (19).
Declaration of interest

The authors declare that there is no conflict of interest that could be perceived as prejudicing the impartiality of the research reported.

\section{Funding}

This work was partially supported by a grant from the 'Fondazione Berlucchi' (Brescia, Italy, call year 2011 to M Volante).

\section{Acknowledgements}

The authors would like to warmly thank Drs Claudia Cavallari and Marco Tampellini for helpful discussion, suggestions, and comments.

\section{References}

1 Mazzaferri EL, Robbins RJ, Spencer A, Braverman LE, Pacini F, Wartofsky L, Haugen BR, Sherman SI, Cooper DS, Braunstein GD et al. A consensus report of the role of serum thyroglobulin as a monitoring method for low-risk patients with papillary thyroid carcinoma. Journal of Clinical Endocrinology and Metabolism 200388 1433-1441. (doi:10.1210/jc.2002-021702)

2 Durante C, Montesano T, Torlontano M, Attard M, Monzani F, Tumino S, Costante G, Meringolo D, Bruno R, Trulli F et al. Papillary thyroid cancer: time course of recurrences during postsurgery surveillance. Journal of Clinical Endocrinology and Metabolism 201398 636-642. (doi:10.1210/jc.2012-3401)

3 Alves GV, Santin AP \& Furlanetto TW. Prognosis of thyroid cancer related to pregnancy: a systematic review. Journal of Thyroid Research 20112011 691719. (doi:10.4061/2011/691719)

4 Moosa M \& Mazzaferri EL. Outcome of differentiated thyroid cancer diagnosed in pregnant women. Journal of Clinical Endocrinology and Metabolism 199782 2862-2866. (doi:10.1210/jcem.82.9.4247)

5 Yasmeen S, Cress R, Romano PS, Xing G, Berger-Chen S, Danielsen B \& Smith LH. Thyroid cancer in pregnancy. International Journal of Gynaecology and Obstetrics 200591 15-20. (doi:10.1016/j.ijgo.2005. 06.022)

6 Herzon FS, Morris DM, Segal MN, Rauch G \& Parnell T. Coexistent thyroid cancer and pregnancy. Archives of Otolaryngology-Head and Neck Surgery 1994120 1191-1193. (doi:10.1001/archotol.1994. 01880350009002)

7 Vannucchi G, Perrino M, Rossi S, Colombo C, Vicentini L, Dazzi D, Beck-Peccoz P \& Fugazzola L. Clinical and molecular features of differentiated thyroid cancer diagnosed during pregnancy. European Journal of Endocrinology 2010162 145-151. (doi:10.1530/EJE-09-0761)

8 Magri F, Capelli V, Rotondi M, Leporati P, La Manna L, Ruggiero R, Malovini A, Bellazzi R, Villani L \& Chiovato L. Expression of estrogen and androgen receptors in differentiated thyroid cancer: an additional criterion to assess the patient's risk. Endocrine-Related Cancer 201219 463-471. (doi:10.1530/ERC-11-0389)

9 Pacini F, Schlumberger M, Dralle H, Elisei R, Smit JW, Wiersinga W \& European Thyroid Cancer Taskforce. European consensus for the management of patients with differentiated thyroid carcinoma of the follicular epithelium. European Journal of Endocrinology $2006 \mathbf{1 5 4}$ 787-803. (doi:10.1530/eje.1.02158)

10 Hedinger C. In Histological Typing of Thyroid Tumors: WHO International Histological Classification of Tumors, 2nd edn. Berlin-HeidelbergNew York: Springer-Verlag, 1988.

11 UICC. In TNM Classification of Malignant Tumours, 6th edn. New York: Wiley Liss, 2002.

12 Cooper DS, Doherty GM, Haugen BR, Kloos RT, Lee SL, Mandel SJ, Mazzaferri EL, McIver B, Sherman SI \& Tuttle RM. Management 
guidelines for patients with thyroid nodules and differentiated thyroid cancer. Thyroid 200616 109-142. (doi:10.1089/thy.2006.16.109)

13 Chiovato L, Latrofa F, Braverman LE, Pacini F, Capezzone M, Masserini L, Grasso L \& Pinchera A. Disappearance of humoral thyroid autoimmunity after complete removal of thyroid antigens. Annals of Internal Medicine 2003139 346-351. (doi:10.7326/0003-4819-1395_Part_1-200309020-00010)

14 Spencer CA, Takeuchi M, Kazarosyan M, Wang CC, Guttler RB, Singer PA, Fatemi S, LoPresti JS \& Nicoloff JT. Serum thyroglobulin autoantibodies: prevalence, influence on serum thyroglobulin measurement, and prognostic significance in patients with differentiated thyroid carcinoma. Journal of Clinical Endocrinology and Metabolism 199883 1121-1127. (doi:10.1210/jcem.83.4.4683)

15 Volante M, Rapa I, Gandhi M, Bussolati G, Giachino D, Papotti M \& Nikiforov YE. RAS mutations are the predominant molecular alteration in poorly differentiated thyroid carcinomas and bear prognostic impact. Journal of Clinical Endocrinology and Metabolism 200994 4735-4741. (doi:10.1210/jc.2009-1233)

16 Ceppi P, Volante M, Novello S, Rapa I, Danenberg KD, Danenberg PV, Cambieri A, Selvaggi G, Saviozzi S, Calogero R et al. ERCC1 and RRM1 gene expressions but not EGFR are predictive of shorter survival in advanced non-small-cell lung cancer treated with cisplatin and gemcitabine. Annals of Oncology 200617 1818-1825. (doi:10.1093/ annonc/mdl300)

17 Webb RC, Howard RS, Stojadinovic A, Gaitonde DY, Wallace MK, Ahmed J \& Burch HB. The utility of serum thyroglobulin measurement at the time of remnant ablation for predicting disease-free status in patients with differentiated thyroid cancer: a meta-analysis involving 3947 patients. Journal of Clinical Endocrinology and Metabolism 201297 2754-2763. (doi:10.1210/jc.2012-1533)

18 Kim TH, Park YJ, Lim JA, Ahn HY, Lee EK, Lee YJ, Kim KW, Hahn SK, Youn YK, Kim KH et al. The association of the BRAF(V600E) mutation with prognostic factors and poor clinical outcome in papillary thyroid cancer: a meta-analysis. Cancer 2012118 1764-1773. (doi:10.1002/ cncr.26500)

19 Stagnaro-Green A, Abalovich M, Alexander E, Azizi F, Mestman J, Negro R, Nixon A, Pearce EN, Soldin OP, Sullivan S et al. Guidelines of the American Thyroid Association for the diagnosis and management of thyroid disease during pregnancy and postpartum. Thyroid 201121 1081-1125. (doi:10.1089/thy.2011.0087)

Received 4 November 2013

Revised version received 20 January 2014

Accepted 7 February 2014 